\title{
Ichthyological survey on the Yucatan Coastal Corridor (Southern Gulf of Mexico)
}

\author{
Evaluación ictiológica en el Corredor Costero de Yucatán \\ (Sureste del Golfo de México)
}

\author{
Sonia Palacios-Sánchez*, María Eugenia Vega-Cendejas*, Mirella Hernández*
}

\begin{abstract}
It is provided a systematic checklist of the ichthyofauna inhabiting the Yucatan coastal corridor, as part of the Mesoamerican Corridor which connects two of the most important reserves in Yucatan Peninsula Mexico: Celestun and Ria Lagartos. Fish specimens were collected bimonthly, from January 2002 to March 2004, in 24 localities along the coast (140 km). The systematic list includes 94 species belonging to 44 families and 19 orders. The best represented families by species number were Sciaenidae (10), Carangidae (9) and Engraulidae (5). Information about size range, number of specimen per species and zoogeographic affinities are included. The species with the highest occurrence $(100 \%)$ were Harengula jaguana and Trachinotus falcatus. It is confirmed the presence of Rypticus maculatus (Serranidae) in the southern Gulf of Mexico and of three brackish species into the marine environment.
\end{abstract}

Keywords: Biodiversity, Coastal fishes, Gulf of Mexico, Ichthyofauna, Yucatan.

\section{Resumen}

\begin{abstract}
Se presenta un listado sistemático de la ictiofauna que habita el corredor costero de Yucatán, el cual forma parte del Corredor Mesoamericano que conecta dos de las reservas más importantes en la Península de Yucatán (México): Celestún y Ría Lagartos. Los especímenes se colectaron bimensualmente entre enero 2002 a marzo 2004 en 24 sitios a lo largo de los 300 km de costa. El listado sistemático contiene 94 especies incluidas en 44 familias y 19 órdenes. Las familias mejor representadas por número de especies fueron Sciaenidae (10), Carangidae (9) y Engraulidae (5). Para cada especie se incluye el número de ejemplares, intervalo de tallas y afinidades zoogeográficas. Las especies con la mayor ocurrencia (100\%) fueron Harengula jaguana y Trachinotus falcatus. Se confirma la presencia de Rypticus maculatus (Serranidae) en el sureste del Golfo de México y de tres especies salobres en el ambiente marino.
\end{abstract}

Palabras clave: Biodiversidad, Golfo de México, Ictiofauna, Peces costeros, Yucatán.

\section{Introduction}

Taxonomic inventories (or species censuses) are the most elementary data in biogeography, macroecology and conservation biology. They play fundamental roles in the construction of species richness patterns, delineation of species ranges, quantification of extinction risk and prioritization of conservation efforts in hot spot areas (Gaston and Blackburn 2000). It is recognized that this knowledge is the starting point for subsequent studies and analysis for fisheries research, fish management, environmental assessments, as well biogeographic and phylogenetic studies, among others (Karr 1981, Moncayo-Estrada et al. 2006). The basic knowledge of diversity alpha through species discovery and description is mostly complete for some areas of the world and for many families of fishes, but important gaps remain (Eschmeyer et al. 2010). Remarkably, tropical areas that are well known for their diversity have among the lowest completeness of all taxonomic inventories. These data gaps occurred regardless of habitat (Mora et al. 2008).

In Yucatan Peninsula (YP) because of karst con-

* Centro de Investigación y de Estudios Avanzados del IPN. Unidad Mérida, km. 6 Antig. Carr. Progreso. AP 73 Cordemex, Mérida, Yucatán, México, CP 97310. e-mail: maruvega@mda.cinvestav.mx

Date received: May 12, $2014 \quad$ Date approval: Diciembre 16, $2014 \quad$ Associated Editor: Mantilla H. 
ditions, soils are porous with rapid infiltration of rainwater and absence of surface drainage (Southworth 1984). The water flow is mostly underground, where drainage is discharged into the direction of the coast. The absence of rivers and freshwater discharges develop in coastal and, heterogeneous habitats (mangroves, petenes, seagrass) for fishes (Toledo-Ocampo 2005, Yáñez-Arancibia et al. 2009), where marine fish species migrate to and from coastal lagoons and wetlands for feeding, reproduction and protection (Arceo-Carranza et al. 2013). In these environments fish species are represent by freshwater, estuarine, euryhaline and estenohaline marine components, some of them of endemic nature.

Coastal fishes of the northwest coast of YP, are of particular interest because they share fauna elements with the Caribbean Province and Western Tropical Atlantic region; about $6.4 \%$ of fish species recorded in the Gulf of Mexico (GM), are restricted to the southeast subregion (i.e. Cape Rojo, Veracruz; Cape Catoche, Quintana Roo), and although most of them occur in the Caribbean, some are endemic to the YP, like the giant killifish Fundulus grandissimus (Miller 2005).

The YP separates the Caribbean Sea from the GM, with $1,100 \mathrm{~km}$ of coastline. It is the exposed portion of the larger Yucatan Platform, which is composed of carbonate and soluble rock with a shallow depth and an unconfined flat lying karst landscape. The northwestern coast comprises the not protected area with $140 \mathrm{~km}^{2}$ of coastline from El Palmar $\left(21^{\circ} 0.3^{\prime}\right.$ $\left.90^{\circ} 16.0^{\prime}\right)$ to Chabihau ( $\left.21^{\circ} 21.0^{\prime}-89^{\circ} 7.0^{\prime}\right)$. In this biological corridor diverse economic activities are realized, as fishing, aquaculture, tourism and port trade. The predominant climate of this region is the driest of the subhumid type with summer rains and a low proportion of winter precipitation (Batllori et al. 2000).

In general, fish research in the southern GM has been concentrated in the coastal lagoons from Veracruz to Yucatan (Reséndez-Medina 1983, Salvadores-Baledón and Reséndez 1990, Yáñez-Arancibia et al. 1993, Obregón-Barboza et al.1994, Pérez-Hernández and Torres-Orozco 2000, Ayala-Pérez et al. 2003, Vega-Cendejas 2004, García-Hernández et al. 2009, Arceo-Carranza et al. 2010, Palacios-Sánchez y Vega-Cendejas, 2010, Peralta-Meixuero and Vega-Cendejas 2011, Vega-Cendejas et al. 2012,
Caballero-Vázquez and Vega-Cendejas 2012), while littoral species have been little studied. In the particular case of the northwestern coast of the YP, there is a lack of systematic information. The present research provides the first checklist of the coastal biological corridor of the Yucatan (CBCY), whose function is to maintain the physical and biological connection between two of the most important biosphere and ecological reserves of Mexico: Celestun and Ria Lagartos (Smardon 2009).

\section{Materials and methods}

Fish collections were conducted monthly during a biannual period (January 2002-March 2004) in twenty four sites along the coast (Figure 1, Table 1). Temperature and salinity were recorded using a multiparametric probe (Yellow Springs Instrument, model 85). Fish specimens were caught with a beach seine (15 $\mathrm{m}$ long, $2 \mathrm{~m}$ high, $2.5 \mathrm{~cm}$ mesh size), approximately at $30 \mathrm{~m}$ from the coastline (Figure 2). A total of forty meters were trawled, covering an estimated area of $264 \mathrm{~m}^{2}$ with two replicas per station. Specimens were preserved in $15 \%$ formalin solution and subsequently transferred to ethyl alcohol.

Each individual was identified to species, measured (standard length to the nearest $0.1 \mathrm{~cm}$ ), and weighed (to the nearest $0.1 \mathrm{~g}$ ). Species identification was based on several taxonomic sources (Fischer 1978,

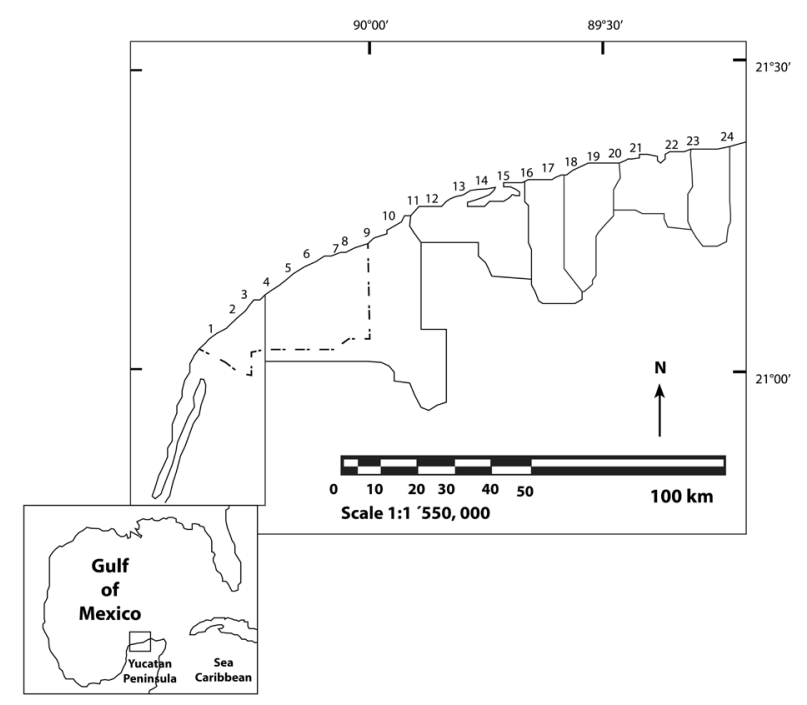

Figure 1. Study area indicating the sampling sites in the northwestern coast of Yucatan Peninsula (Southern Gulf of Mexico). 
Table 1. List of sampling sites in the northwest coast of Yucatan Peninsula with their code number

\begin{tabular}{|c|c|c|c|c|}
\hline & Station & Position & $\% \mathrm{~N}$ & $\mathbf{S}$ \\
\hline El Palmar & (1) Faro M & $21^{\circ} 3.49^{\prime}, 90^{\circ} 15.93^{\prime}$ & 4.4 & 27 \\
\hline Reserve & (2) Faro I-M & $21^{\circ} 3.05^{\prime}, 90^{\circ} 16.46^{\prime}$ & 21.8 & 38 \\
\hline \multirow[t]{3}{*}{ Western zone } & (3) Cahuich & $21^{\circ} 4.46^{\prime}, 90^{\circ} 15.02^{\prime}$ & 2.0 & 21 \\
\hline & (4) Punta Pantera & $21^{\circ} 5.36^{\prime}, 90^{\circ} 12.71$ & 13.5 & 33 \\
\hline & (5) La tabla & $21^{\circ} 6.80^{\prime}, 90^{\circ} 10.81$ & 5.3 & 35 \\
\hline El Palmar Reserve & (6) Punta Elefante & $21^{\circ} 8.04^{\prime}, 90^{\circ} 9.55^{\prime}$ & 10.3 & 36 \\
\hline \multirow[t]{5}{*}{ Eastern zone (EPR) } & (7) Punta Piedra & $21^{\circ} 9.46^{\prime}, 90^{\circ} 5.74^{\prime}$ & 1.1 & 33 \\
\hline & (8) Yaxantún & $21^{\circ} 9.69^{\prime}, 90^{\circ} 4.31^{\prime}$ & 1.6 & 36 \\
\hline & (9) Pecis-M & $21^{\circ} 10.65^{\prime}, 89^{\circ} 59.91^{\prime}$ & 1.2 & 31 \\
\hline & (10) Bocana-M & $21^{\circ} 13.73^{\prime}, 89^{\circ} 54.35^{\prime}$ & 7.8 & 37 \\
\hline & (11) Chuburná & $21^{\circ} 15.40^{\prime}, 89^{\circ} 49.34^{\prime}$ & 2.5 & 27 \\
\hline \multirow[t]{4}{*}{ Progreso (PG) } & (12) Chelem & $21^{\circ} 16.02^{\prime}, 89^{\circ} 46.78^{\prime}$ & 0.8 & 16 \\
\hline & (13) Progreso & $21^{\circ} 17.25^{\prime}, 89^{\circ} 40.10^{\prime}$ & 4.4 & 37 \\
\hline & (14) Chicxulub & $21^{\circ} 14.55^{\prime}, 89^{\circ} 37.73^{\prime}$ & 1.6 & 25 \\
\hline & (15) Uaymitun & $21^{\circ} 17.99^{\prime}, 89^{\circ} 35.13^{\prime}$ & 0.8 & 33 \\
\hline \multirow{4}{*}{ Ixil (IX) } & (16) San Bruno II & $21^{\circ} 18.72^{\prime}, 8^{\circ} 32.33^{\prime}$ & 2.4 & 40 \\
\hline & (17) San Benito & $21^{\circ} 19.41^{\prime}, 89^{\circ} 29.04^{\prime}$ & 1.2 & 36 \\
\hline & (18) San Bruno & $21^{\circ} 19.56^{\prime}, 89^{\circ} 26.55^{\prime}$ & 1.0 & 30 \\
\hline & (19) Telchac I & $21^{\circ} 20.61^{\prime}, 89^{\circ} 18.33^{\prime}$ & 1.3 & 24 \\
\hline \multirow[t]{2}{*}{ Telchac (TC) } & (20) Telchac II & $21^{\circ} 20.61^{\prime}, 89^{\circ} 16.70^{\prime}$ & 3.2 & 33 \\
\hline & (21) San Crisanto I & $21^{\circ} 20.92^{\prime}, 89^{\circ} 13.64^{\prime}$ & 2.6 & 37 \\
\hline \multirow[t]{2}{*}{ Sinanche (SC) } & (22) San Crisanto II & $21^{\circ} 21.27^{\prime}, 89^{\circ} 10.56^{\prime}$ & 1.3 & 36 \\
\hline & (23) Chabihau I & $21^{\circ} 21.44^{\prime}, 89^{\circ} 7.62^{\prime}$ & 5.9 & 34 \\
\hline Yobaín (YB) & (24) Chabihau II & $21^{\circ} 21.47^{\prime}, 89^{\circ} 7.13^{\prime}$ & 2.1 & 32 \\
\hline
\end{tabular}

Geographic coordinates (Latitude N, Longitude W), relative numerical abundance $(\% \mathrm{~N})$ and species richness $(\mathrm{S})$ are specified.

Whitehead 1985, Hoese and Moore 1998, McEachran and Fechhelm 1998, 2005, Schmitter-Soto 1998, Castro-Aguirre et al. 1999, Carpenter 2002a, 2002b), and systematic classification according to Nelson (2006). The name, author and description year were checked against Eschmeyer (2015). A representative sample of each species was deposited and catalogued in the ichthyological collection of CINVESTAV-IPN, Merida (YUC.PEC.084.0999 key).

The list includes information about the number of individuals collected by species, size ranges of the specimens expressed in $\mathrm{cm}$ of standard length and their occurrence frequency in the region. The ichthyo-geographic classification was assigned according to Briggs (1974) and considering the information published on the geographic distribution of the species: West Atlantic (WA), Circumtropical (CT), Gulf of Mexico (GM), Amphiamerican (AA) Amphiatlantic (AT) and Yucatan Peninsula (YP). The comparisons of maximum sizes recorded in previous studies were performed considering the Fishbase website (Froese and Pauly 2015).

\section{Results}

As a tropical region, the study area showed little variations in temperature and salinity, with average values of $27.02( \pm 2.31)^{\circ} \mathrm{C}$ and $35.84( \pm 1.71)$, respectively. A total of 94 species included in 81 genera, 44 families and 19 orders were sampled during the study. From this total, the Eslasmobranchs (Chondrichthyes) were represented by three orders, six families, six genera and six species, while Teleosts (Actinopterigii) by 16 orders, 38 families, 75 genera, and 88 species (Table 2). The best represented families were Sciaenidae (10 species), Carangidae (9) and Engraulidae (5). The highest relative numerical abundance (21\%) and species richness (38 species) were recorded in localities from the Palmar Reserve, located near a Biosphere Reserve (Celestun), while the lowest values of these ecological parameters were found in localities from Progreso (Table 1). Twenty species ( $80 \%$ equivalent to the total catch recorded), were found in more than $80 \%$ from all localities $(>75 \%$ occurrence frequency); within these, we have the en- 


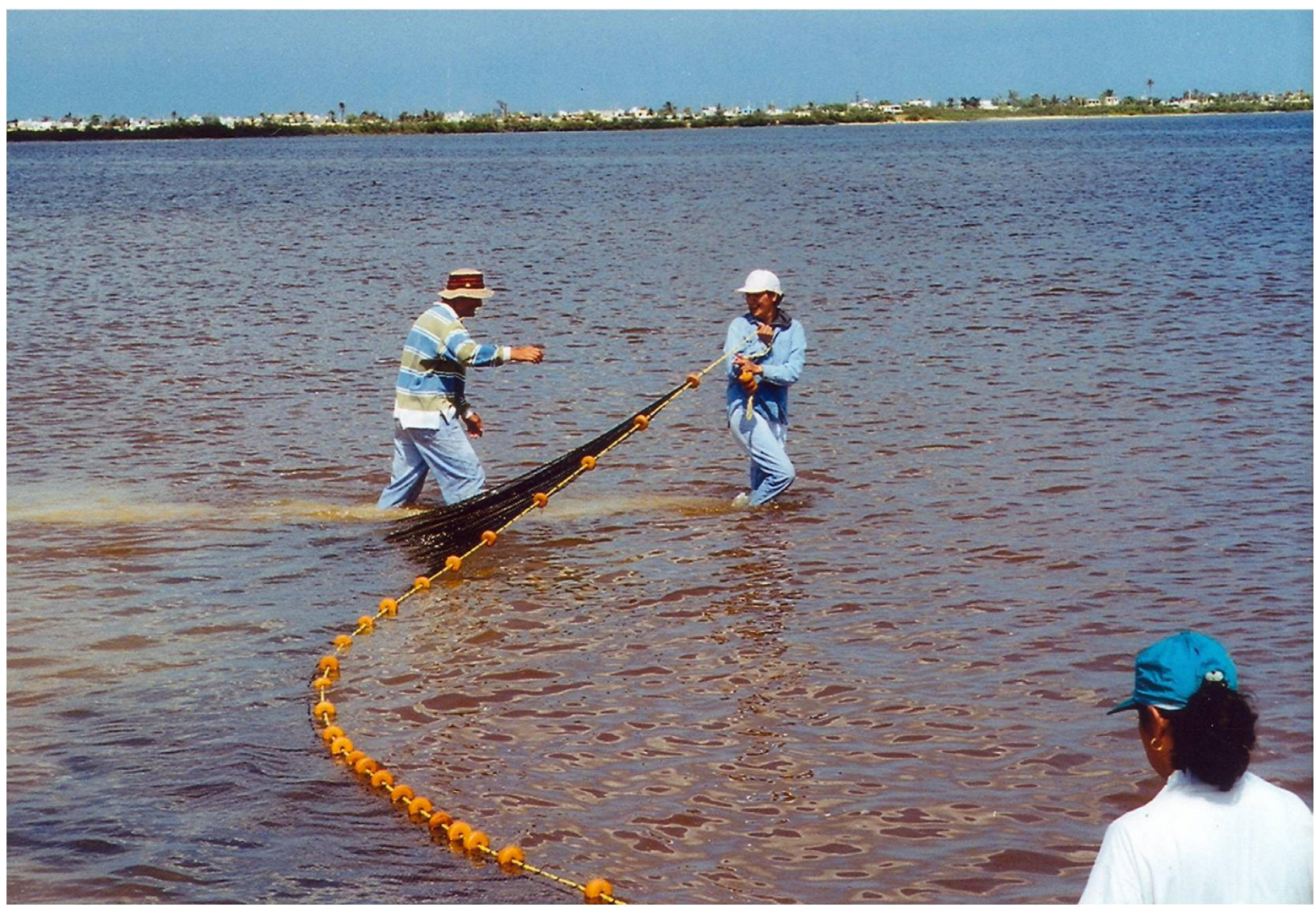

Figure 2. Collecting fish with a beach seine in northwestern coast of Yucatan Peninsula.

Table 2. Total numbers of the Orders, Families, Genera and Species recorded in the northwestern coast of Yucatan Peninsula

\begin{tabular}{|c|c|c|c|c|}
\hline Classes & Orders & Families & Genera & Species \\
\hline \multirow{3}{*}{ Chondrichthyes } & Torpediniformes & 1 & 1 & 1 \\
\hline & Rajiformes & 1 & 1 & 1 \\
\hline & Myliobatiformes & 4 & 4 & 4 \\
\hline Total & 3 & 6 & 6 & 6 \\
\hline \multirow{16}{*}{ Actinopterygii } & Elopiformes & 1 & 1 & 1 \\
\hline & Albuliformes & 1 & 17 & 1 \\
\hline & Clupeiformes & 2 & 3 & 8 \\
\hline & Siluriformes & 1 & 2 & 2 \\
\hline & Aulopiformes & 1 & 1 & 1 \\
\hline & Ophidiiformes & 1 & 1 & 1 \\
\hline & Batrachoidiformes & 1 & 1 & 1 \\
\hline & Lophiiformes & 2 & 2 & 2 \\
\hline & Mugiliformes & 1 & 1 & 2 \\
\hline & Beloniformes & 2 & 4 & 7 \\
\hline & Cyprinodontiformes & 2 & 2 & 3 \\
\hline & Gasterosteifomes & 1 & 1 & 3 \\
\hline & Scorpaeniformes & 1 & 1 & 3 \\
\hline & Perciformes & 14 & 29 & 41 \\
\hline & Pleuronectiformes & 3 & 4 & 4 \\
\hline & Tetraodontiformes & 4 & 5 & 8 \\
\hline Total & 16 & 38 & 75 & 88 \\
\hline Totales & 19 & 44 & 81 & 94 \\
\hline
\end{tabular}


graulids (Anchoa mitchilli, Anchoa hepsetus), gerrids Eucinostomus argenteus, Eucinostomus gula), catfish (Sciades felis) and checkered puffer (Sphoeroides testudineus). The species with the highest occurrence (100\%) were Harengula jaguana and Trachinotus falcatus. By contrast 35 species showed an occasional occurrence $(<2 \%)$ (Table 3$)$.

The fish fauna of the northwest coast of YP was characterized by a mixture of tropical, subtropical and temperate-warm species with 73 exclusive from west Atlantic, five Circumtropical, eight Amphiatlantic, two Amphiamerican, one Amphiatlantic/Amphiamerican, two endemic from the GM (McEachran and Fechhelm 2005), and three from YP (Miller 2005) (Figure 3). The curatorial information obtained in this research is part of the National Information System on Biodiversity (SNIB-CONABIO), which website (www.conabio.gob.mx) is available for consultation.

\section{Discussion}

Biological corridors are fundamental strategies for biodiversity conservation in fragmented landscapes. However, little evidence exists about their functionality or the population viability of its species on long term especially for the tropics (González-Maya et al. 2013). The CBCY, is spatially located among two biosphere reserves, which maintain the biological diversity in the unprotected area.

The report of fish species at specific sites is probably the most basic data in ecology (Gaston and Blackburn 2000). Such records are often used to calculate the number of species occurring at each site or as a source of information with which to extrapolate the extent of species occurrence and their area of occupancy. There is a general agreement that without species-level data, basic processes such as energy fluxes, biological interactions, evolutionary history, habitat specificity, and biogeographic distributions cannot be studied. Identification of exotic species and the establishment of reserves are not possible without reliable information on species distributions (Grassle and Stocks 1999). Our incomplete knowledge about the current fish distribution in tropical areas also raises concern upon the effectiveness of conservation efforts (Mora et al. 2008).

One of the species recorded in the northwest coast of YP, is within the category of critically endangered (Narcine bancroftii), two of them near threatened (Rhinobatos lentiginosus, Aetobatus narinari) (IUCN 2015), and three are reported with a size range greater than previously recorded by Froese and Pauly (2015)

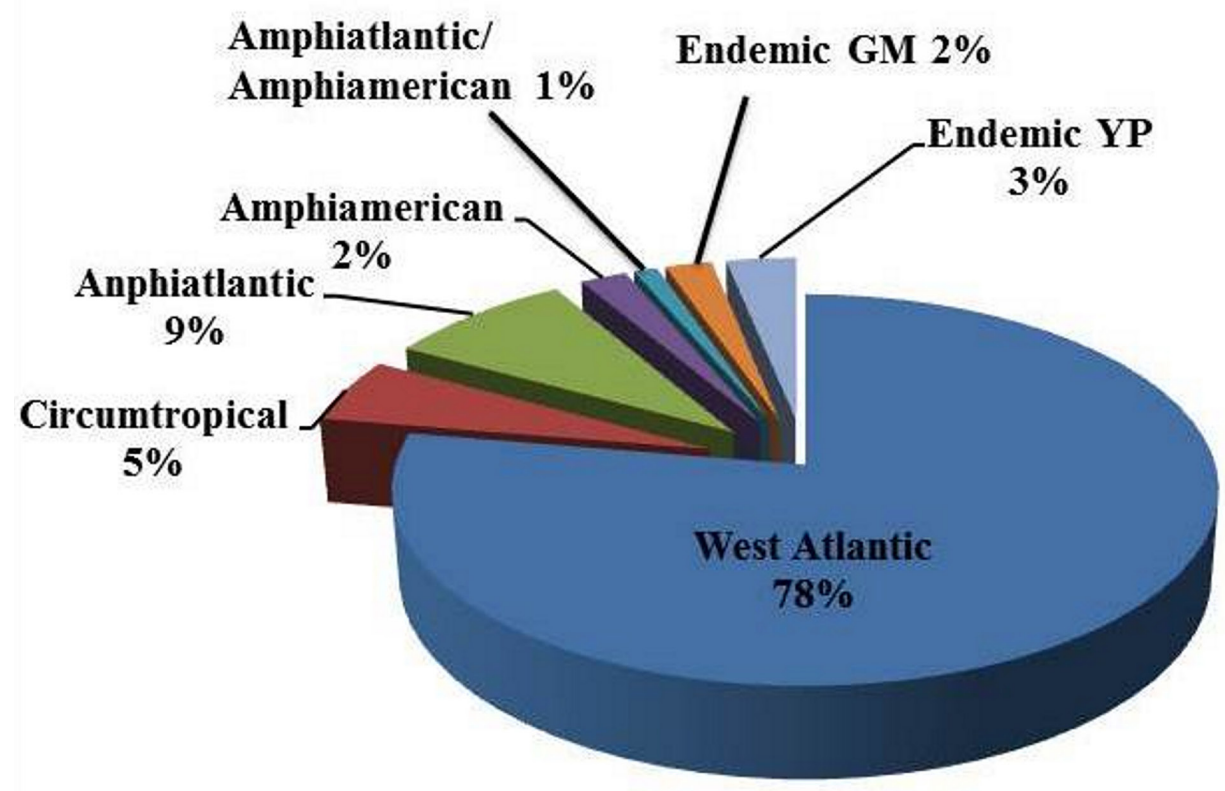

Figure 3. Relative contribution of ichthyogeographic categories recorded in the northwestern coast of Yucatan Peninsula, based on Briggs (1974). 
Table 3. Fish checklist of the northwestern coast of Yucatan Peninsula (Southern Gulf of Mexico)

\begin{tabular}{|c|c|c|c|c|}
\hline Taxa & Sampling sites & SL & $\mathrm{n}>1,[\mathrm{OF}]$ & D \\
\hline \multicolumn{5}{|l|}{ Chondrichthyes } \\
\hline \multirow{2}{*}{\multicolumn{5}{|c|}{$\begin{array}{l}\text { I. Torpediniformes } \\
\text { Narcinidae }\end{array}$}} \\
\hline & & & & \\
\hline 1.Narcine bancroftii (Griffith \& Smith 1834) ${ }^{\mathrm{CE}}$ & 8 & 25.0 & [4.2] & WA \\
\hline \multicolumn{5}{|l|}{ II. Rajiformes } \\
\hline Rhinobatidae & & & & \\
\hline 2. Rhinobatos lentiginosus Garman $1880^{\mathrm{NT}}$ & 23,24 & 28.5-34.9 & 8 [8.3] & WA \\
\hline \multirow{2}{*}{\multicolumn{5}{|c|}{$\begin{array}{l}\text { III. Myliobatiformes } \\
\text { Urotryaonidae }\end{array}$}} \\
\hline & & & & \\
\hline 3. Urobatis jamaicensis (Cuvier 1816) & $6-9,14-16,21,23$ & $12.5-29.9$ & $11[37.5]$ & WA \\
\hline Dasyatidae & & & & \\
\hline $\begin{array}{l}\text { 4. Dasyatis americana Hildebrand \& Schroeder } 1928 \\
\text { Gymnuridae }\end{array}$ & 23,24 & $44.2-56.7$ & 2 [8.3] & WA \\
\hline 5. Gymnura micrura (Bloch \& Schneider 1801) & $1-11,13,16-18,21-24$ & 13.0-21.7 & 68 [79.2] & WA \\
\hline Myliobatidae & & & & \\
\hline 6. Aetobatus narinari (Euphrasen 1790) ${ }^{\mathrm{NT}}$ & 24 & 128.0 & [4.2] & CT \\
\hline \multicolumn{5}{|l|}{ Actinopterygii } \\
\hline \multirow{2}{*}{\multicolumn{5}{|c|}{$\begin{array}{l}\text { IV. Elopiformes } \\
\text { Elopidae }\end{array}$}} \\
\hline & & & & \\
\hline 7. Elops saurus Linnaeus 1766 & $2,6-10,15-16,19,21$ & $19.5-31.5$ & $27[41.7]$ & WA \\
\hline \multicolumn{5}{|l|}{ V. Albuliformes } \\
\hline Albulidae & & & & \\
\hline 8. Albula vulpes (Linnaeus 1758) & $7,8,10-11,16,19,21-22$ & $3.2-14.8$ & 23 [33.3] & CT \\
\hline \multicolumn{5}{|l|}{ VI. Clupeiformes } \\
\hline \multicolumn{5}{|l|}{ Engraulidae } \\
\hline 9. Anchoa cubana (Poey 1868) & $1-2,6-7,9$ & $2.0-5.9$ & $493[20.8]$ & WA \\
\hline 10. Anchoa hepsetus (Linnaeus 1758) & $1-2,5-11,13-14,16-21$ & $2.7-10.5$ & $3972[70.8]$ & WA \\
\hline 11. Anchoa lamprotaenia Hildebrand $1943^{*}$ & $1-2,4-24$ & $2.6-12.8$ & $883[95.8]$ & WA \\
\hline 12. Anchoa lyolepis (Evermann \& Marsh 1900) & $1-2,4,6-7,13,19$ & $3.5-6.3$ & $263[29.2]$ & WA \\
\hline 13. Anchoa mitchilli (Valenciennes 1848) & $1-2,4-22,24$ & $2.3-10.1$ & 1914 [91.7] & WA \\
\hline \multicolumn{5}{|l|}{ Clupeidae } \\
\hline 14. Harengula humeralis (Cuvier 1829) $)^{\lrcorner}$ & $14-16,18$ & 2.3-3.1 & $22[16.7]$ & WA \\
\hline 15. Harengula jaguana Poey $1865^{\star}$ & $1-24$ & $1.87-25.0$ & $5470[100.0]$ & WA \\
\hline 16. Opisthonema oglinum (Lesueur 1818) & $4-8,10-11,13-14,23,19-20$ & & & \\
\hline \multicolumn{5}{|l|}{ VII. Siluriformes } \\
\hline \multicolumn{5}{|l|}{ Ariidae } \\
\hline 17. Sciades felis (Linnaeus 1766) & $1,3-5,6-11,13-24$ & $4.0-26.3$ & 1458 [91.7] & WA \\
\hline 18. Bagre marinus (Mitchill 1815) & $1,3-4,9-10,13-14,18,20$ & $7.2-15.3$ & $26[37.5]$ & WA \\
\hline \multicolumn{5}{|l|}{ VIII. Aulopiformes } \\
\hline \multicolumn{5}{|l|}{ Synodontidae } \\
\hline 19. Synodus foetens (Linnaeus 1766) & $1-10,13,15,18,20-24$ & $3.9-24.5$ & $45[75.0]$ & WA \\
\hline \multirow{2}{*}{\multicolumn{5}{|c|}{ IX. Ophidiformes }} \\
\hline Bythitidae & & & & \\
\hline 20. Gunterichthys longipenis Dawson 1966 & 16 & 6.2 & [4.2] & GM \\
\hline \multicolumn{5}{|l|}{ X. Batrachoidiformes } \\
\hline \multicolumn{5}{|l|}{ Batrachoididae } \\
\hline 21. Opsanus beta (Goode \& Bean 1880) & $7,16,17,21$ & 4.5-10.4 & $23[16.7]$ & WA \\
\hline \multicolumn{5}{|l|}{ XI. Lophiiformes } \\
\hline Antennariidae & & & & \\
\hline 22. Histrio histrio (Linnaeus 1758$)^{\lrcorner}$ & 15 & 5.5 & [4.2] & CT \\
\hline $\begin{array}{l}\text { Ogcocephalidae } \\
23 \text { Ogcocephalus cubifrons (Richardson 1836) }\end{array}$ & 23 & 21.2 & {$[4.2]$} & WA \\
\hline XII. Mugiliformes & & & & \\
\hline Mugilidae & & & & \\
\hline
\end{tabular}


Table 3. Fish checklist of the northwestern coast of Yucatan Peninsula (Southern Gulf of Mexico) (continuation)

\begin{tabular}{|c|c|c|c|c|}
\hline Taxa & Sampling sites & SL & $n>1,[O F]$ & D \\
\hline 24. Mugil curema Valenciennes 1836 & $2,4,7-8,10-11,13,24$ & $2.0-23.9$ & 14 [33.3] & AA/AT \\
\hline 25. Mugil trichodon Poey $1875^{\lrcorner}$ & $9,13,16,20,21-22,24$ & $2.1-15.3$ & 50 [29.2] & WA \\
\hline \multicolumn{5}{|l|}{ XIII. Beloniformes } \\
\hline \multicolumn{5}{|l|}{ Hemiramphidae } \\
\hline 26. Chriodorus atherinoides Goode \& Bean 1882 & $1,4,7,10,12,17-18,22-24$ & $4.2-18.5$ & $42[41.7]$ & WA \\
\hline 27. Hyporhamphus meeki Banford \& Collette $1993^{*}$ & * 21 & 19.3 & [4.2] & WA \\
\hline 28.Hyporhamphus unifasciatus (Ranzani 1841) & $1-2,4-8,11-13,15-18,20-24$ & $6.2-25.5$ & $162[79.2]$ & $A A$ \\
\hline \multicolumn{5}{|l|}{ Belonidae } \\
\hline 29. Strongylura marina (Walbaum 1792) & 12,17 & $9.0-28.0$ & $2[8.3]$ & WA \\
\hline 30. Strongylura notata (Poey 1860) & $1-7,12-18,21-22,24$ & $4.7-46.0$ & $118[70.8]$ & WA \\
\hline 31. Strongylura timucu (Walbaum 1792) & $5,8,16,21-22,24$ & $7.2-39.3$ & $11[25.0]$ & WA \\
\hline 32. Tylosurus crocodilus (Péron \& Lesueur 1821) & 2,3 & $4.5-75.0$ & $3[4.2]$ & $\mathrm{CT}$ \\
\hline \multicolumn{5}{|l|}{ XIV. Cyprinodontiformes } \\
\hline \multicolumn{5}{|l|}{ Fundulidae } \\
\hline 33. Fundulus grandissimus Hubbs $1936^{\mathrm{E}}$ & 10 & 8.7 & {$[4.2]$} & YP \\
\hline $\begin{array}{l}\text { 34. Fundulus persimilis Miller 1955E, }{ }^{*} \\
\text { Cyprinodontidae }\end{array}$ & $4-5,9-10,17,21$ & $2.6-18.5$ & $14[25]$ & YP \\
\hline 35. Floridichthys polyommus Hubbs 1936 & $3,5,20$ & $1.7-8.0$ & $3[8.3]$ & YP \\
\hline \multicolumn{5}{|l|}{ XV. Gasterosteiformes } \\
\hline \multicolumn{5}{|l|}{ Syngnathidae } \\
\hline 36. Syngnathus floridae (Jordan \& Gilbert 1882) & 17 & 11.9 & [4.2] & WA \\
\hline 37. Syngnathus louisi & 15 & 11.0 & [4.2] & WA \\
\hline 38. Syngnathus pelagicus Linnaeus 1758 & 4 & 7.5 & {$[4.2]$} & WA \\
\hline \multicolumn{5}{|l|}{ XVI. Scorpaeniformes } \\
\hline \multicolumn{5}{|l|}{ Triglidae } \\
\hline 39. Prionotus scitulus Jordan \& Gilbert 1882 & $9,22-24$ & 3.3-11.1 & $7[16.7]$ & WA \\
\hline 40. Prionotus tribulus Cuvier 1829 & $5,16,18,2$ & -14.3 & $35[20.8]$ & WA \\
\hline \multicolumn{5}{|l|}{ XVII. Perciformes } \\
\hline \multicolumn{5}{|l|}{ Centropomidae } \\
\hline 41. Centropomus undecimalis (Bloch 1792) ${ }^{\lrcorner}$ & 19 & $27.1-32.2$ & $2[4.2]$ & \\
\hline CINV-NEC 2436; () & & & & WA \\
\hline \multicolumn{5}{|l|}{ Serranidae } \\
\hline 42. Diplectrum bivittatum (Valenciennes 1828$)^{\lrcorner}$ & 20 & 13.9 & [4.2] & WA \\
\hline 43. Rypticus maculatus Holbrook $1855^{\lrcorner}$ & $5-18,20$ & $6.4-8.9$ & 10 [20.8] & WA \\
\hline \multicolumn{5}{|l|}{ Carangidae } \\
\hline 44. Caranx bartholomaei Cuvier 1833 & 22 & 6.8 & [4.2] & WA \\
\hline 45. Caranx crysos (Mitchill 1815) & $2,15-16,22-23$ & $9.7-13.0$ & 5 [20.8] & AT \\
\hline 46. Caranx hippos (Linnaeus 1766) & 6,13 & $3.6-17.3$ & $3[8.3]$ & AT \\
\hline 47. Caranx latus Agassiz 1831 & $4-6,16-17,20-21,24$ & $7.0-14.9$ & 16 [33.3] & AT \\
\hline 48. Oligoplites saurus (Bloch \& Schneider 1801) & $2,4-6,8-11,13,17,21-22,24$ & $4.2-23.8$ & $22[50.0]$ & AA \\
\hline 49. Selene vomer (Linnaeus 1758) & $4-8,10-11,13,16-17,20$ & $1.8-12.5$ & $26[45.8]$ & WA \\
\hline 50. Trachinotus carolinus (Linnaeus 1766) & $1-2,4-14,16-20,22-24$ & $1.8-11.4$ & $114[87.5]$ & WA \\
\hline 51. Trachinotus falcatus (Linnaeus 1758) & $1-24$ & $1.4-14.5$ & $561[100.0]$ & WA \\
\hline $\begin{array}{l}\text { 52. Trachinotus goodei Jordan \& Evermann } 1896 \\
\text { Lutjanidae }\end{array}$ & $1-2,5,10-11,13-15,17,20,22$ & $2.9-15.3$ & $25[45.8]$ & WA \\
\hline 53. Lutjanus analis (Cuvier 1828) & 21 & 6.6 & [4.2] & WA \\
\hline 54. Lutjanus griseus (Linnaeus 1758) & $2-5,10,13,15,17-19,22-24$ & $4.8-16.3$ & $37[54.2]$ & WA \\
\hline \multicolumn{5}{|l|}{ Lobotidae } \\
\hline 55. Lobotes surinamensis (Bloch 1790) & 18,13 & $5.2-19.1$ & $2[8.3]$ & CT \\
\hline \multicolumn{5}{|l|}{ Gerreidae } \\
\hline 56. Eucinostomus argenteus Baird \& Girard 1855 & $2-11,13-24$ & $1.6-19.0$ & 401 [91.7] & WA \\
\hline 57. Eucinostomus gula (Quoy \& Gaimard 1824) & $2-17,19-24$ & $2.6-9.1$ & $26[91.7]$ & WA \\
\hline 58. Eucinostomus harengulus Goode \& Bean, 1879 & $2,6,10,18,22$ & $1.6-10.2$ & $25[20.8]$ & WA \\
\hline 59. Eugerres plumieri (Cuvier 1830) & 5,15 & $15.3-19.0$ & $4[8.3]$ & WA \\
\hline
\end{tabular}


Table 3. Fish checklist of the northwestern coast of Yucatan Peninsula (Southern Gulf of Mexico) (continuation)

\begin{tabular}{|c|c|c|c|c|}
\hline Taxa & Sampling sites & SL & $n>1$, [OF] & D \\
\hline \multicolumn{5}{|l|}{ Haemulidae } \\
\hline 60. Anisotremus virginicus (Linnaeus 1758) & 14 & 8.0 & [4.2] & WA \\
\hline 61. Haemulon flavolineatum (Desmarest 1823) & 2 & $7.5-15.7$ & $2[4.2]$ & WA \\
\hline 62. Haemulon plumierii (Lacepède 1801) & 11 & 2.5 & [4.2] & WA \\
\hline $\begin{array}{l}\text { 63. Orthopristis chrysoptera (Linnaeus 1766) } \\
\text { Sparidae }\end{array}$ & $2,9-10,13,15,20-21,23-24$ & $4.2-20.5$ & $19[37.5]$ & WA \\
\hline 64. Archosargus probatocephalus (Walbaum 1792) & $16-17$ & $12.0-13.6$ & $3[8.3]$ & WA \\
\hline 65. Archosargus rhomboidalis (Linnaeus 1758) & $1-18,20-23$ & 2.7-20.2 & 151, [91.7] & WA \\
\hline $\begin{array}{l}\text { 66. Lagodon rhomboides (Linnaeus 1766) } \\
\text { Polynemidae }\end{array}$ & $1-13,15,17-24$ & $4.7-13.0$ & 259 [91.7] & WA \\
\hline $\begin{array}{l}\text { 67. Polydactylus virginicus (Linnaeus 1758) } \\
\text { Sciaenidae }\end{array}$ & $1,10,13,19-21$ & $4.4-10.0$ & $8[25.0]$ & WA \\
\hline 68. Bairdiella chrysoura (Lacepède 1802) & $1-3,5,7-9,11-15,17-21,23-24$ & $3.3-17.0$ & 140 [79.2] & WA \\
\hline 69. Bairdiella ronchus (Cuvier 1830) & 8,7 & 13.6-15.6 & $2[8.3]$ & WA \\
\hline 70. Corvula sanctaeluciae Jordan 1890 & $8,17-18,21$ & $4.9-17.5$ & $8[16.7]$ & WA \\
\hline 71. Cynoscion arenarius Ginsburg 1930 & $1-11,13-15,17-21$ & 2.6-20.9 & $105[79.2]$ & GM \\
\hline 72. Cynoscion nebulosus (Cuvier 1830) & $1-3,5,7,13,15,17$ & $3.5-18.5$ & 17 [33.3] & WA \\
\hline 73. Menticirrhus americanus (Linnaeus 1758) & $1-23$ & 2.1-16.2 & 129 [95.8] & WA \\
\hline 74. Menticirrhus littoralis (Holbrook 1847) & $1,4-22,24$ & $1.7-15.7$ & $90[87.5]$ & WA \\
\hline 75. Menticirrhus saxatilis (Bloch \& Schneider 1801) & $1,2,4-17,19-23$ & 2.1-19.0 & $90[87.5]$ & WA \\
\hline 76. Micropogonias undulatus (Linnaeus 1766) & $8,13,15$ & $3.0-9.3$ & $3[12.5]$ & WA \\
\hline 77. Umbrina coroides Cuvier 1830 & 20 & 11.6 & {$[4.2]$} & WA \\
\hline \multicolumn{5}{|l|}{ Kyphosidae } \\
\hline \multirow{2}{*}{\multicolumn{5}{|c|}{ Scaridae }} \\
\hline & & & & \\
\hline 79. Cryptotomus roseus Cope 1871 & 5 & 4.7 & $4,[4.2]$ & WA \\
\hline \multirow{2}{*}{\multicolumn{5}{|c|}{ Ephippidae }} \\
\hline & & & & \\
\hline $\begin{array}{l}\text { 81. Chaetodipterus faber (Broussonet 1782) } \\
\text { Sphyraenidae }\end{array}$ & $6,8,13-14,16,21-22$ & $2.8-13.8$ & $21[29.2]$ & WA \\
\hline 82. Sphyraena barracuda (Edwards 1771) & 23 & 25.8 & [4.2] & AT \\
\hline \multicolumn{5}{|l|}{ XVIII. Pleuronectiformes } \\
\hline \multicolumn{5}{|l|}{ Paralichthyidae } \\
\hline 83. Citharichthys macrops Dresel 1885 & $8,10,13,15-16,18,22,24$ & $3.6-10.4$ & 8 [33.3] & WA \\
\hline $\begin{array}{l}\text { 84. Paralichthys albigutta Jordan \& Gilbert } 1882 \\
\text { Achiridae }\end{array}$ & 6,10 & $8.5-28.0$ & $2[8.3]$ & WA \\
\hline 85. Achirus lineatus (Linnaeus 1758) & $11,19,21,23,24$ & $7.5-16.9$ & $11[20.8]$ & WA \\
\hline Cynoglossidae & & & & \\
\hline 86. Symphurus plagiusa (Linnaeus 1766) & $2,9,19-24$ & $6.0-14.4$ & 16 [33.3] & WA \\
\hline \multicolumn{5}{|l|}{ XIX. Tetraodontiformes } \\
\hline \multicolumn{5}{|l|}{ Monacanthidae } \\
\hline 87. Stephanolepis hispidus (Linnaeus 1766) & 16 & 3.3-3.8 & $4[4.2]$ & AT \\
\hline $\begin{array}{l}\text { 88. Stephanolepis setifer (Bennett 1831) } \\
\text { Ostraciidae }\end{array}$ & $2,4,9$ & $3.6-6.5$ & $6[12.5]$ & WA \\
\hline 89. Acanthostracion quadricornis (Linnaeus 1758) & $3,4,6,10,14,16,22$ & $12.9-21.5$ & 17 [29.2] & AT \\
\hline 90. Lagocephalus laevigatus (Linnaeus 1766) & 8 & 9.6 & [4.2] & AT \\
\hline 91. Sphoeroides nephelus (Goode \& Bean 1882) & $1,3,5,7,9-1016-17,20-22$ & 2.3-9.6 & $15[45.8]$ & WA \\
\hline 92. Sphoeroides spengleri (Bloch 1785) & $6,8,16-17,22-23$ & $3.8-12.7$ & $24[25.0]$ & WA \\
\hline 93. Sphoeroides testudineus (Linnaeus 1758) & $1-11,13-17,19-24$ & $3.5-28.0$ & $132[91.7]$ & WA \\
\hline Diodontidae & & & & \\
\hline 94. Chilomycterus schoepfii (Walbaum 1792) & 15,24 & 3.3-7.5 & $22[8.3]$ & WA \\
\hline
\end{tabular}

*Species with a maximum length greater than previously recorded (Froese and Pauly 2013). J: juveniles CE: Species critically endangered NT: Species near threatened E: Species endemic. Sampling sites, size range (cm) as standard length (SL), number of individuals (n), occurrence frequency [OF] and distribution (D), are indicated for each species: west Atlantic (WA), circumtropical (CT), Gulf of Mexico (GM), amphiamerican (AA) amphiatlantic (AT) and Yucatan Peninsula (YP). Sampling site code numbers are from Table1. 


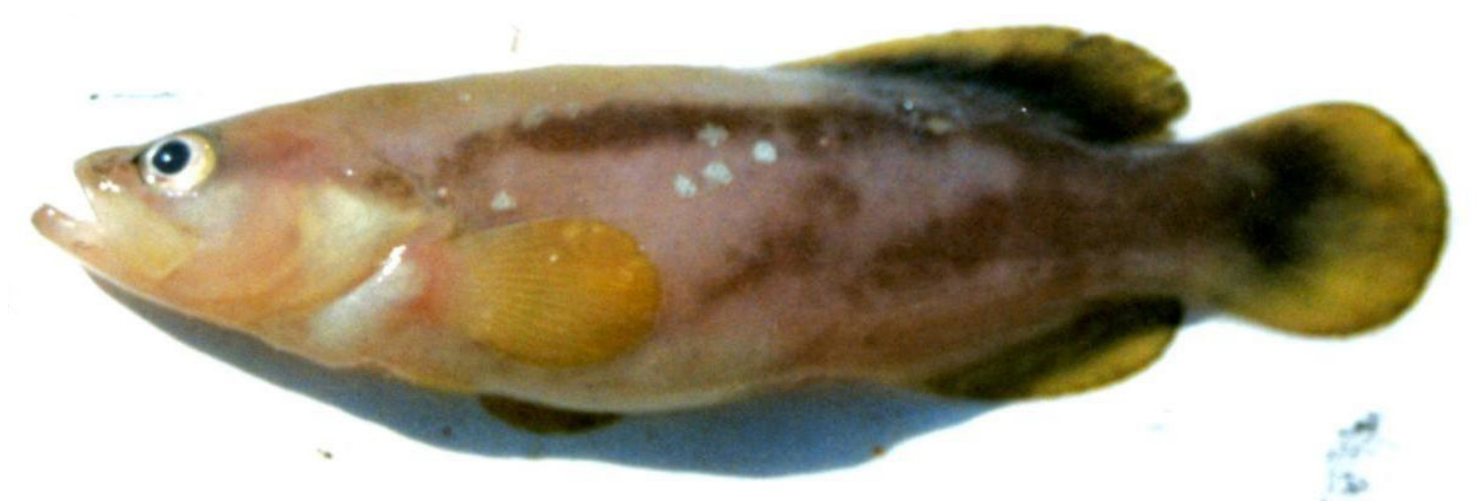

Figure 4. Rypticus maculatus (Serranidae) recorded in the northwestern coast of Yucatan Peninsula.

(Table 3). From this set of species, is confirmed the presence of Rypticus maculatus (Serranidae) (Figure 4), as in the southern GM has only been reported in the Campeche Bank (Carpenter 2002a, McEachran and Fechhelm 1998, McEachran and Fechhelm 2005).

According to the proposed ecological classification by Castro-Aguirre et al. (1999), $46.8 \%$ of the recorded species belong to the stenohaline marine component, and $44.7 \%$ to marine eurihaline, so most of these species ( $82 \%$ ) have been reported in coastal lagoons of the northeast and northwest YP coast (Vega-Cendejas 2004, Arceo-Carranza et al. 2010, Peralta-Meixuero and Vega-Cendejas 2011, Caballero-Vázquez and Vega-Cendejas 2012). The occurrence in the marine environment of typical freshwater and brackish species of the northwest coast of Yucatan like Fundulus grandissimus, Fundulus persimilis and Floridichthys polyommus indicate a clear relation to the hydrological conditions prevailing on the YP coast: 1) direct access of seawater with the marsh through breaks of the land barrier between them, and 2) underwater springs which discharge fresh water from the aquifers to sea water, mainly during the rainy season (Herrera-Silveira et al. 2004, Aranda-Cirerol et al. 2006). Both processes coupled with the high salinity tolerance of these species, may explain their occasional occurrence in the coastal marine area, and stretch the high proportion of the marine fish composition recorded in the area (>90\%).

Though most of the recorded species have a wide distribution in the Mexican Caribbean (62), Netherlands Antilles (45), Bahamas (43), Bermudas (39) and in northwest of the Gulf of Mexico (48) (Guitart 1974, Smith et al. 1975, Robins and Ray 1986;
Böhlke and Chaplin 1993, Darovec 1995, Hoese and Moore 1998, Smith-Vaniz et al. 1999, Schmitter-Soto et al. 2000), their diversity and distribution patterns are related to nearshore habitat heterogeneity and the bioecological dependence of many marine species to coastal lagoons and wetlands. The present checklist is a taxonomic inventory of an area that is important for its biodiversity and highlights the value of the coastal systems within the biological strategies of marine fish species. The lack of taxonomic inventories can introduce substantial mistakes in assessing diversity patterns, and raise concerns over the effectiveness of conservation strategies. While specific data are lacking, fishing activities, both artisanal and commercial in nature are generally intense and most often unregulated in shallow inshore waters. Changes in community structure and abundance of even the most common, well-known species add a sense of urgency to measurement of the present composition and limits of marine life to evaluate human impacts and making management decisions.

\section{Acknowledgements}

To Consejo Nacional de Ciencia y Tecnología, México (CONACyT) by a graduate scholarship to Sonia E. Palacios Sánchez; to Comisión Nacional de Biodiversidad, México (CONABIO) for financial support for research Project (CONABIO-027) and to Alex Acosta, Walter Canto, Víctor Castillo, Oscar Reyes and Eloy Gil† for field assistance. Special thanks are given to Dr. Luis Capurro Filograsso for review and comments on the manuscript. 


\section{Literature cited}

Aranda-Cirerol N, Herrera-Silveira JA, Comín, FA. 2006. Nutrient water quality in a tropical coastal zone with groundwater discharge, northwest Yucatan, Mexico. Est Coast Shelf Sci. 68 (3-4): 445-54.

Arceo-Carranza D, Vega-Cendejas ME, Montero-Muñoz JL, Hernández de SM. 2010. Influencia del hábitat en las asociaciones nictimerales de peces en una laguna costera tropical. Rev Mex Biodiversidad. 81: 823-37.

Arceo-Carranza D, Vega-Cendejas ME, Hernández de SM. 2013. Day and night trophic variations of dominant fish species in a lagoon influenced by freshwater seeps. J Fish Biol. 82: 54-68.

Ayala-Pérez LA, Ramos-Miranda J, Flores-Hernández D. 2003. La comunidad de peces de la Laguna de Términos: estructura actual comparada. Rev Biol Trop. 51 (3-4): 783-94.

Batllori E, Dickinson F, García A, Martín M, González I, Villasuso M, Febles JL. 2000. Socioecological regions of the Yucatan Peninsula. pp. 33-53. In: Lutzm W, Prieto L, Sanderson W (eds.). Population, development, and environment on the Yucatán Peninsula: From Ancient Maya to 2030. Laxenburg: International Institute for Applied Systems Analysis.

Böhlke JE, Chaplin CC. 1993. Fishes of the Bahamas and Adjacent Tropical Waters. 2nd ed. Austin: University of Texas Press. 771 pp.

Briggs JC. 1974. Marine zoogeography. New York: McGrawHill. 475 pp.

Caballero-Vázquez JA, Vega-Cendejas ME. 2012. Spatial patterns of diversity at local and regional scales in a tropical lagoon. Neotrop Ichthyol. 10 (1): 99-108.

Carpenter KE (ed). 2002a. The living marine resources of the Western Central Atlantic. Bony fishes part 1 (Acipenseridae to Grammatidae). Volume 2. FAO Species Identification Guide for Fishery Purposes and American Society of Ichthyologists and Herpetologists. Special publication $\mathrm{N}^{\circ} 5$. Rome: FAO; pp. 601-1374.

Carpenter KE (ed). 2002b. The living marine resources of the Western Central Atlantic. Bony fishes part 2 (Opistognathidae to Molidae), sea turtles and marine mammals. Volume 3. FAO Species Identification Guide for Fishery Purposes and American Society of Ichthyologists and Herpetologists Special Publication $\mathrm{N}^{\circ}$ 5. Rome: FAO; pp. 1375-2127.

Castro-Aguirre JL, Espinosa-Pérez H, Schmitter-Soto JJ. 1999. Ictiofauna estuarino-lagunar y vicaria de México. México, DF: Ed. Limusa; 711 pp.

Darovec JE. 1995. Checklist and local distribution analyses of fishes from the Hourglass Cruises. Florida Department Natural Research. Memoirs of the Hourglass Cruises. 4 (1): $1-130$.

Eschmeyer WN (ed). 2015. Catalog of fishes on line. San Francisco: California Academy of Sciences. Captured, 29 April 2015. Electronic Database accessible at http://research. calacademy.org/research/ichthyology/catalog/

Eschmeyer NW, Fricke RJ, Fong D, Polack DA. 2010. Marine fish diversity: history of knowledge and discovery (Pisces). Zootaxa. 2525: 19-50.
Fischer W. 1978. Species identification sheets for fisheries purposes. Western Central Atlantic. Vols. I-V. Rome: FAO.

Froese R, Pauly D. 2015. FishBase. Captured on 29 April, 2013. Electronic Database accessible at http://www.fishbase.org

García-Hernández V, Ordóñez-López U, Hernández-Vázquez T, Álvarez-Cadena J. 2009. Fish larvae and juveniles checklist (Pisces) from the northern Yucatan Peninsula, Mexico, with 39 new records for the region. Rev Mex Biodiversidad. 80: 85-94.

Gaston KJ, Blackburn TM. 2000. Pattern and process in macroecology. Oxford: Blackwell Science; 377 pp.

González-Maya FJ, Vela-Vargas I, Pineda-Guerrero A. 2013. Valuation method to evaluate the viability of biological corridors for mammals: a preliminary analysis of San JuanLa Selva corridor in Costa Rica. Rev Biodivers Neotrop. 3 (1): 30-6.

Grassle JF, Stocks KI. 1999. A global ocean biogeographic information system (OBIS) for the census of marine life. Oceanography. 12 (3): 12-4.

Guitart DJ. 1974. Sinopsis de los peces marinos de Cuba. Vols. I-IV. La Habana: Academia de Ciencias de Cuba.

Herrera-Silveira JA, Comin FA, Aranda-Cirerol N, Troccoli L, Capurro L. 2004. Coastal waters quality assessment in the Yucatan Peninsula: management implications. Ocean Coast Manage. 47: 625-39.

Hoese HD, Moore RH. 1998. Fishes of the Gulf of Mexico: Texas, Louisiana and Adjacent Waters. Texas: Texas A \& M University Press; 422 pp.

IUCN. 2015. Red List of Threatened Species. Version 2014.3. Captured on 29 April 2015. Accessible at http://www. iucnredlist.org.

Karr JR. 1981. Assessment of biotic integrity using fish communities. Fisheries. 6 (6): 21-7.

McEachran JD, Fechhelm JD. 1998. Fishes of the Gulf of Mexico. Volume 1, Myxiniformes to Gasterosteiformes. Austin: University of Texas Press; 1112 pp.

McEachran JD, Fechhelm JD. 2005. Fishes of the Gulf of Mexico. Volume II, Scorpaeniformes to Tetraodontiformes. Austin: University of Texas Press; 1004 pp.

Miller RR. 2005. Freshwater fishes of Mexico. Chicago, London: The University of Chicago Press; $490 \mathrm{pp}$.

Moncayo-Estrada R, Castro-Aguirre JL, De La Cruz-Agüero J. 2006. Lista sistemática de la ictiofauna de Bahía de Banderas, México. Rev Mex Biodiversidad. 77: 67-80.

Mora C, Tittensor DP, Myers RA. 2008. The completeness of taxonomic inventories for describing the global diversity and distribution of marine fishes. Proc Royal Soc. 275: 149-55.

Nelson JS. 2006. Fishes of the world. Hoboken: John Wiley \& Sons.

Obregón-Barboza H, Contreras-Balderas S, Lozano-Vilano ML. 1994. The fishes of northern and central Veracruz, México. Hydrobiologia. 286: 79-95.

Palacios-Sánchez S, Vega-Cendejas ME. 2010. Cambios alimenticios en tres especies de Sphoeroides (Tetraodontiformes: Tetraodontidae) posterior al huracán Isidoro en Bocana de la Carbonera, Sureste del Golfo de México. Rev Biol Trop. 58: 1223-35.

Peralta-Meixueiro MA, Vega-Cendejas ME. 2011. Spatial and 
temporal structure of fish assemblages in a hyperhaline coastal system: Ría Lagartos, Mexico. Neotrop Ichthyol. 9 (3): 673-82.

Pérez-Hernández MA, Torres-Orozco BR. 2000. Evaluación de la riqueza de especies de peces en las lagunas costeras mexicanas: estudio de un caso en el Golfo de México. Rev Biol Trop. 48 (2-3): 425-38.

Reséndez-Medina A. 1983. Hidrología e ictiofauna de la Laguna de Zontecomapan, Veracruz, Mexico. Ana Inst Biol Univ Nac Autón Mex. Serie Zoología. 53 (1): 385-417.

Robins CR, Ray C.1986. A field guide to Atlantic coast fishes of North America. Boston: Houghton Mifflin Co; 354 pp.

Salvadores-Baledón M, Reséndez A. 1990. Modificaciones en la composición ictiofaunística del sistema lagunar El Carmen-Machona, Tabasco, por la apertura de la boca panteones. Univ Ciencia. 7 (14): 5-13.

Schmitter-Soto JJ. 1998. Catálogo de los peces continentales de Quintana Roo. México: ECOSUR; 239 pp.

Schmitter-Soto JJ, Vásquez-Yeomanes L, Aguilar-Perera A, Uriel-Mondragón C, Caballero-Vázquez A. 2000. Lista de peces marinos del Caribe mexicano. Ana Inst Cienc Mar Limno Univ Nac Autón Mex. Serie Zoología. 71 (2): 143-77.

Smardon R. 2009. Sustaining the World's Wetlands. London: Springer Science. 326 pp.

Smith GB, Austin HM, Bortone SA, Hastings RW, Ogren LH. 1975. Fishes of the Florida middle ground with comments on ecology and zoogeography. St Petersburg: Florida Marine Research Publications $N^{\circ}$ 9. 14 pp.

Smith-Vaniz WF, Collette BB, Luckhurst BE. 1999. Fishes of Bermuda: History, zoogeography, annotated checklist, and identification keys. Washington, DC: American Society of Ichthyologists and Herpetologists; 424 pp.

Southworth CS. 1984. Structural and hydrogeologic applications of remote sensing data, eastern Yucatan Peninsula, Mexi- co. pp. 59-64. In: Beck N (ed). Sinkholes: Their geology, engineering and environmental impact. Proceedings 1st. Orlando: Multi-disciplinary Conference on Sinkholes.

Toledo-Ocampo A. 2005. Marco conceptual: caracterización ambiental del Golfo de México; pp. 25-52. In: Botello AV, Rendón-Von Osten J, Gold-Bouchot G, Agraz-Hernández C. (ed.). Golfo de México contaminación e impacto ambiental: diagnóstico y tendencias. México, DF: Universidad Autónoma de Campeche, Universidad Nacional Autónoma de México, Instituto Nacional de Biología; 696 pp.

Vega-Cendejas ME. 2004. Ictiofauna de la reserva de la biosfera Celestún, Yucatán: una contribución al conocimiento de su biodiversidad. Ana Inst Biol Univ Nac Auton Mex. Serie Zoología. 75 (1): 193-206.

Vega-Cendejas ME, Hernández de SM, Arceo D. 2012. Length-weight relationships for selected fish species from a coastal lagoon influenced by freshwater seeps: Yucatan peninsula, Mexico. J App Ichthyol. 28 (1): 140-2.

Whitehead PJP. 1985. Clupeoid fishes of the world. An annotated and illustrated catalogue of the herrings, sardines, pilchards, sprats, anchovies and wolf-herrings. Rome: FAO; Pt. 1; 303 pp.

Yáñez-Arancibia A, Lara-Domínguez AL, Day JJr. 1993. Interactions between mangrove and sea-grass habitats mediated by nekton assemblages: coupling of primary and secondary production. Hydrobiologia. 264: 1-12.

Yáñez-Arancibia A, Ramírez-Gordillo JJ, Day JW, Yoskowitz DW. 2009. Environmental sustainability of economic trends in the Gulf of Mexico: What is the limit for Mexican coastal development? pp. 82-104. In: Tunnell JW, Felder DJJr, Earle SA. (ed.). Gulf of Mexico origin, waters and biota. Vol. 2. Ocean and coastal economy. Corpus Christ: Harte Research Institute for Gulf of Mexico Studies Series. Texas AM. Univ. Press; 110 pp. 